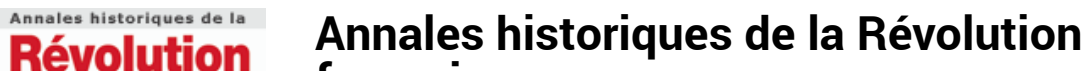

française française

364 | avril-juin 2011

Varia

\section{French Historical Studies}

Jean-Pierre Gross

\section{(2) OpenEdition}

Journals

Édition électronique

URL : https://journals.openedition.org/ahrf/12074

DOI : 10.4000/ahrf.12074

ISSN : $1952-403 X$

Éditeur :

Armand Colin, Société des études robespierristes

Édition imprimée

Date de publication : 1 juin 2011

Pagination : 260-263

ISSN : 0003-4436

Référence électronique

Jean-Pierre Gross, «French Historical Studies ». Annales historiques de la Révolution française [En ligne], 364 | avril-juin 2011, mis en ligne le 31 août 2011, consulté le 23 avril 2022. URL : http:// journals.openedition.org/ahrf/12074 ; DOI : https://doi.org/10.4000/ahrf.12074

Ce document a été généré automatiquement le 23 avril 2022.

Tous droits réservés 


\title{
French Historical Studies
}

\author{
Jean-Pierre Gross
}

\section{RÉFÉRENCE}

French Historical Studies, Duke University Press, Durham, N. C., Special Issue : 89: Then and Now, Volume 32, Number 4, Fall 2009, p. 527-706

1 La revue French Historical Studies a publié à l'automne 2009 un numéro rétrospectif sur le thème "1989: vingt ans après ", consacré à l'historiographie de la Révolution française depuis le Bicentenaire. Y ont contribué une douzaine d'auteurs, dont plus de la moitié, réunie en forum, dresse un bilan des nouvelles tendances enregistrées, les autres ayant rédigé de courtes études monographiques. Dans le premier groupe on retrouve les historiens David Andress, Laurent Dubois, Carla Hesse, Lynn Hunt, Colin Jones, Jean-Clément Martin, Sophia Rosenfeld et J. B. Shank; dans le deuxième, Malcolm Crook, Philippe R. Girard, Mary Ashburn Miller et Timothy Tackett. Les conclusions qui se dégagent des réflexions du forum sont plutôt réservées, dans la mesure où l'année 1989 avait engendré une sorte d'euphorie, à la suite d'ébranlements majeurs: massacres de la place Tian An-Men, chute du mur de Berlin, puis effondrement de l'empire soviétique, disparition des démocraties populaires, fin effective du maoïsme, fin de la guerre froide. Pendant ce temps, le révisionnisme, privilégiant une lecture libérale de l'histoire, continuait à faire des ravages et remettait en cause le bloc monolithique de la Révolution française fondatrice d'un nouvel ordre mondial, la remplaçant par des révolutions au pluriel, des changements à facettes multiples étalés sur plusieurs décennies. L'historiographie récente, assagie et assainie, en est ressortie circonspecte, éclectique, pluraliste et pragmatique.

2 Faut-il s'en réjouir ou s'en lamenter ? Colin Jones, plutôt déconcerté, nous rappelle que Simon Schama, l'auteur de Citizens, le bestseller de l'année du Bicentenaire, avait résumé la Terreur de 1794 comme étant " 1789 agrémenté d'un plus grand nombre de cadavres ", résumant ainsi l'approche révisionniste et postrévisionniste, dépouillant la Révolution de son aura de fons et origo de la modernité et conférant aux Lumières, selon 
l'interprétation habermasienne toujours en vogue, une importance accrue. Colin Jones relève avec surprise le manque de mondialisation entre historiens de cultures intellectuelles différentes. Tandis que Sarah Maza aux États-Unis dénonce le mythe de la bourgeoisie, Jean-Pierre Jessenne en France cherche à nuancer la voie qui mène «vers un ordre bourgeois». Tandis que les Américains épousent la "French Theory» postmoderne et poststructuraliste à la Derrida, les historiens français lui tournent résolument le dos. Ne faudrait-il pas redécouvrir l'économie politique et placer l'égalité et la justice sociale au centre de nos préoccupations, s'interroge Jones, si nous voulons redonner à la Révolution française le rôle central, aujourd'hui occulté, qu'elle a joué dans l'invention de la modernité ?

3 À la lumière d'ouvrages récents sur le patriotisme de la noblesse, la sociabilité des milieux privilégiés ou la duplicité du couple royal, David Andress en déduit que l'histoire réelle est infiniment plus complexe que le mythe. Ne faudrait-il pas commencer par se débarrasser de celui-ci? Car ce qui passait pour la Révolution française jusqu'en 1989 n'était-ce pas au bout du compte un mythe? Aux yeux d'Andress, l'historiographie française de la Révolution semble avoir profité des vingt dernières années pour se remettre de sa propre histoire et, en cessant d'être l'enjeu de débats politiques stériles, peut aujourd'hui servir de tremplin à des conjectures enrichississantes sur la nature même de la modernité qu'elle aurait engendrée, selon le vœu même exprimé par Colin Jones.

4 Carla Hesse nous apprend qu'une nouvelle génération d'historiens emprunte une troisième voie pour sortir de l'impasse du Bicentenaire. Dans une contribution originale dont le titre peut surprendre, "The New Jacobins », elle se défend de vouloir réhabiliter les historiens marxistes que François Furet en 1989 condamnait pour leurs préjugés "néojacobins ", mais cherche au contraire à remettre la Révolution à sa place dans la trajectoire historique du républicanisme français, prenant comme repère l'existence d'un centre républicain radical, issu du jacobinisme, de cet extrême-centre invisible mais omniprésent postulé par Pierre Serna. Carla Hesse se réfère ainsi à l'émergence d'institutions républicaines sous la Convention et se déclare solidaire des chercheurs qui y décernent les signes avant-coureurs d'un État de droit, tels le souci de la légalité et la volonté de placer la Loi au centre même de la réflexion politique, là où le révisionnisme néolibéral n'a perçu que terreur ou utopie.

5 Les vingt dernières années ont privilégié, outre-atlantique, les études sur l'émancipation, celle des femmes exclues de l'arène politique et celle des esclaves noirs dans les colonies françaises. Plusieurs historiennes adeptes des gender studies se sont attaquées à la toute-puissance du mythe pour découvrir, en dépit de la misogynie contemporaine, les indices d'un féminisme en marche sous la Révolution. Lynn Hunt, qui s'est récemment penché sur l'éthique universaliste des droits humains pour y détecter les ferments d'une telle libération progressive, ou potentielle, de la femme, se contente ici d'évoquer l'expérience révolutionnaire, et notamment la violence, à travers les estampes de la période révolutionnaire, qui se sont multipliées de manière exponentielle durant la décennie 1789 à 1799 et qui constituent un filon à peine exploité. Il n'en reste pas moins que les paradigmes marxiste et néotoquevillien ayant été sabordés, rien de semblable jusqu'à présent ne les a remplacés: l'histoire révolutionnaire lui semble désormais orpheline, privée de paradigmes.

6 Quant à la dimension coloniale, Laurent Dubois redonne vigueur à la notion d'une révolution atlantique, différente de celle proposée jadis par Palmer et Godechot, dans la 
mesure où elle est a priori antillaise et française. Depuis le livre de C. L. R. James sur Toussaint Louverture, publié en 1963 et intitulé The Black Jacobins, longue est la lignée d'études consacrées à Saint-Domingue et à la révolution haïtienne, aux débats passionnés qui se déroulèrent en métropole autour de l'esclavage et la politique coloniale, ainsi qu'à l'impact de la traite des noirs sur les ports atlantiques français. Dubois souligne que la fièvre insurrectionnelle eut une incidence dans toute la région des Caraïbes, depuis la Guyane jusqu'à Santo Domingo, Cuba et la Jamaïque, la Martinique et la Guadeloupe. C'est dans ce contexte que se situe l'étude parallèle de Philippe Girard sur la politique coloniale du Premier Consul Bonaparte et l'expédition de Leclerc à Saint-Domingue, sur l'équivoque qui entoure la loi du 20 mai 1802, dont l'application tout en nuances fut déléguée à Leclerc, puis à Rochambeau. Mais malgré l'effroyable gâchis dans lequel sombrèrent les opérations militaires, Girard souligne que l'esclavage n'y fut jamais rétabli et quand la révolution bascula en une guerre de libération et que Dessalines y proclama l'indépendance, c'est le statut de cultivateurs à demi affranchis institué par Louverture qui prévalut à Haïti.

C'est dans le cadre des Cent Jours, en avril et mai 1815, que Malcolm Crook poursuit sa chronique des élections de la période révolutionnaire et napoléonienne, mettant l'accent sur le vote populaire destiné à ratifier l'acte additionnel de 1815 et les élections des collèges départementaux à la Chambre des Représentants. Bien que le nombre des votants ne fût guère supérieur à un quart de l'électorat, le processus même de consultation électorale contribua fortement à lancer la légende de Napoléon, champion du peuple, promoteur des libertés, défenseur de l'acquis révolutionnaire et de l'honneur de la Nation, victime du royalisme face à la répression qui devait suivre la seconde restauration des Bourbons. En 1815, l'empereur des Cent Jours s'est réinventé comme personnage radical héritier de la Révolution française, représentant du républicanisme autant que de la monarchie héréditaire. Le fait que la pratique électorale avait été ravivée et étendue jusqu'au niveau municipal, conféra à cette osmose une auréole de souveraineté citoyenne que l'Empire avait au contraire érodée. Ainsi naquit le "bonapartisme démocratique », fondé dans l'exercice du suffrage et appelé à un rôle déterminant à partir de 1848 .

8 Timothy Tackett continue à rechercher l'émergence d'une mentalité révolutionnaire sous l'Ancien Régime. Il présente ici les itinéraires de cinq hommes étudiés à travers leur correspondance et leurs journaux intimes écrits pendant les dix à vingt années qui précédèrent la Révolution: Félix Faulcon, Pierre Vergniaud, Gilbert Romme, Nicolas Ruault et Adrien-Joseph Colson. Essai de microhistoire qui démontre l'absence de valeurs culturelles ou de vision idéologique claire, susceptibles de révéler, avant 1787 et la première assemblée des notables, le moindre engagement politique indicatif d'une vocation révolutionnaire. Dans son livre Becoming a Revolutionary, publié en 1996, Tackett avait conclu que le choc psychologique de l'année 1789 chez les Constituants a compté plus que leur formation ou leurs lectures antérieures. Là où nous divergeons un tant soit peu avec le portrait qu'il brosse du jeune Gilbert Romme, c'est au sujet de son éveil socio-économique : celui-ci se situe à nos yeux en 1774-1775, quand Romme a 25 ans et suit de près le débat entre Turgot et Necker relatif à la liberté du commerce des grains. Prélude à la guerre des farines, cette querelle lui fera découvrir les dangers du laisser-faire et il se rangera résolument du côté de Necker qui défend les intérêts des petits consommateurs. L'éveil politique de Romme, en revanche, fut tardif et n'est perceptible qu'à partir d'avril 1789 : mais il fut fulgurant. Assidu aux séances des États généraux, puis de l'Assemblée constituante, il découvrit alors l'éthique des droits de 
l'homme et du citoyen, qui devait lui servir de repère tout au long de sa carrière de député à la Législative et à la Convention. Ce fut pour lui une véritable illumination.

L'explosion de joie qui accompagne cette révélation est symptomatique du caractère émotif de l'engagement révolutionnaire. L'article de Mary Ashburn Miller est consacré à la métaphore du volcan dans le langage de cette époque charnière. Elle constate qu'au début de la Révolution, l'éruption volcanique était le symbole de la force déchaînée et incontrôlée, alors que pour une brève période qui correspond au temps de la Terreur, elle va symboliser la passion patriotique et la vertu républicaine. Le langage devient ainsi le reflet de la forte teneur émotionnelle des passions vécues. Or, l'étude des émotions est devenu un champ largement exploité à présent par des chercheurs attentifs aux manifestations de la psychologie révolutionnaire. Lynn Hunt en 1992 était la première à se pencher sur l'inconscient politique et avait emprunté le thème du «roman familial» à Sigmund Freud. Au dire de Sophia Rosenfeld, l'expérience révolutionnaire se prête à l'analyse, elle révèle la complexité psychologique de l'homme, isolé ou dans la foule, en proie à la frénésie collective des assemblées. Tendance innovative et prometteuse à ses yeux, qui pourrait permettre à la recherche historique de sortir de l'ornière où elle se trouve. L'étude du soi (self en anglais) à une époque où le sentiment a revêtu autant d'importance que la raison, pourrait nous permettre de déterminer comment la sensibilité a pu façonner l'action et la réaction (agency), et décrypter ainsi les soubresauts et les revirements inattendus de la Révolution. L'anxiété, la peur, voire la panique, peuvent mieux expliquer la nature des choix retenus et des actes accomplis en 1793-1794, considérés jusqu'à présent comme découlant de décisions rationnelles ou idéologiques. La contre-révolution fait son retour en scène comme mouvement né de la psychologie de la peur, à la même enseigne que les contre-lumières et l'antiphilosophie. L'effroi analysé par Antoine de Baecque ou Sophie Wahnich, ainsi que la spirale émotive de la suspicion et de l'incompréhension explicitée par William Reddy ou Darrin McMahon, peuvent être de précieux indicateurs.

Il incombe à Jean-Clément Martin (le seul chercheur à apporter une contribution en langue française) de nous offrir un résumé succinct de l'éclectisme régnant, qui oblige à des relectures, privilégie l'étude des particularités régionales et sociales aux dépens de la "grande politique", et englobe une pluralité d'approches, psychologiques, anthropologiques, interdisciplinaires ou comparatistes. Allusion aux études relatives aux révolutions survenues en Amérique latine, qui démontrent que les modèles appliqués n'étaient pas réservés à la seule France: telle, par exemple, la "guerre à mort» menée un moment par Bolívar. Martin souligne l'importance accrue de l'historiographie anglo-américaine devenue hégémonique par sa variété, comme par le nombre des intervenants. Il en conclut sur une note optimiste : la Révolution française, vue dans ses multiples dimensions, "possède une actualité, que les historiens et historiennes doivent revendiquer avec une force nouvelle pour reconquérir une place perdue dans nos sociétés ». 